\title{
АДАПТАЦИЯ ОПРОСНИКА Е.Т. ХИГГИНСА ПО ДИАГНОСТИКЕ ФОКУСА РЕГУЛЯЦИИ НА РУССКОЯЗЫЧНОЙ ВЫБОРКЕ
}

\author{
В.А. ГЕРШКОВИЧ ${ }^{\mathrm{a}}$ Н.В. МОРОШКИНА \\ А.Д. НАСЛЕДОВ
}

${ }^{a}$ Санкт-Петербургский государственный университет, 199034, Россия, Санкт-Петербург, Университетская наб., д. 7/9

\section{Резюме}

В статье представлены результаты адаптации опросника Т. Хиггинса по диагностике фокуса регуляции (Regulatory Focus Questionnaire, RFQ) на русскоязычной выборке. В соответствии с теорией фокуса регуляции целенаправленное поведение регулируется двумя различными мотивационными системами (системами саморегуляции) - фокусом продвижения (promotion) и фокусом профилактики (prevention), которые подразумевают качественно различные средства достижения желаемых состояний. Система продвижения связана с переживанием удовольствия от наличия позитивных исходов и неудовольствия от их отсутствия, а система профилактики связана с переживанием удовольствия от отсутствия негативных исходов (потерь) и неудовольствия от их наличия. В процедуре адаптации опросника приняли участие 536 (в возрасте от 17 до 23 лет) человек, из них 158 - на пилотажном этапе, 308 - на основном этапе исследования. В процессе адаптации опросника выполнен прямой и обратный перевод опросника, проведены проверки: факторной структуры опросника с помощью конфирматорного факторного анализа (КФА), внутренней согласованности шкал опросника, ретестовой надежности. Проверка валидности опросника проводилась с помощью изучения влияния доминирующего фокуса регуляции на подверженность классическим когнитивным искажениям при принятии решений. Результаты применения КФА показали сохранение двухфакторной ортогональной структуры опросника $(\mathrm{CMIN}=52.580 ; \mathrm{df}=39 ; p=0.072 ; \mathrm{GFI}=0.971 ; \mathrm{CFI}=0.957 ; \mathrm{RMSEA}=0.034$; Pclose $=0.884)$, шкалы методики обладают достаточно высокой надежностью по внутренней согласованности их пунктов ( $\alpha$ Кронбаха для шкалы «Фокус продвижения» 0.77 и для шкалы «Фокус профилактики» - 0.78), по ретестовой надежности. Количество вопросов, вошедших в русскоязычную версию, соответствует оригинальной версии опросника. Проведенные исследования свидетельствуют в пользу конструктной валидности шкал. Опросник может быть использован для людей в возрасте от 17 до 23 лет. В статье приведены текст опросника, инструкции и ключ.

Ключевые слова: опросник по диагностике фокуса регуляции, теория фокуса регуляции, фокус продвижения, фокус профилактики, мотивация достижения. 
В настоящее время в зарубежных исследованиях все бо́льшую популярность набирает теория фокуса регуляции (regulatory focus), предложенная T. Хиггинсом в 1997 г. (Higgins, 1997). Эта теория является развитием классической модели мотивации достижения (Atkinson, 1964). Согласно теории мотивации достижения, новая задача, с которой сталкивается человек, вызывает чувства, связанные с выполнением предыдущих заданий. Например, для людей с субъективной историей успеха новая задача достижения вызывает чувство гордости. Это чувство провоцирует реакции предвосхищения будущей цели и направляет поведение на ее достижение (мотивация достижения успеха). С другой стороны, для людей с субъективной историей неудачи новая задача вызывает чувство стыда, и это чувство, в свою очередь, приводит к появлению стратегий, связанных с избеганием достижения цели (мотивация избегания неудачи). В развиваемой Т. Хиггинсом теории фокуса регуляции предполагается, что сами стратегии достижения цели также могут быть различными в зависимости от того, какова была история достижения успеха (см. рисунок 1). Фактически теория фокуса регуляции - это теория о том, как люди достигают поставленных ими целей. Основываясь на том, что люди в основном мотивированы либо на достижение удовольствия, либо, наоборот, на избегание неудовольствия, Т. Хиггинс предполагает, что сами эти цели могут быть репрезентированы совершенно по-разному в зависимости от того, какая система саморегуляции активирована у человека.

Рисунок 1

Связь понятий мотивация достижения/избегания неудач и фокус продвижения/профилактики (Molden et al., 2008, p. 171)

Мотивация на достижение успеха



Фокус продвижения

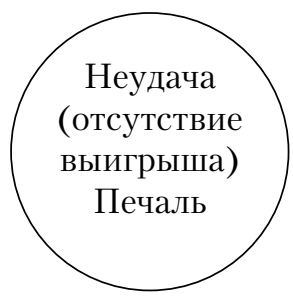



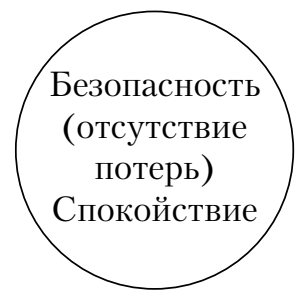

Фокус профилактики

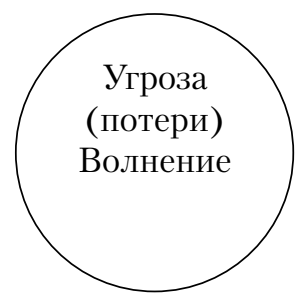

Мотивация на избегание неудачи 
В соответствии с теорией фокуса регуляции (Higgins, 1997, 1998) целенаправленное поведение регулируется двумя различными мотивационными системами (системами саморегуляции) - фокусом продвижения (promotion) и фокусом профилактики (prevention), которые подразумевают качественно различные средства достижения желаемых состояний. В случае работы фокуса продвижения люди представляют цель как надежду и вдохновение, в случае фокуса профилактики - как обязанность или долг. Подобные репрезентации целей приводят соответственно к тому, что люди предпочитают либо более рискованные стратегии поведения (eagerness means), либо стратегии бдительности (vigilance means) и профилактики ошибок для достижения поставленных целей. Система продвижения связана с переживанием удовольствия от наличия позитивных исходов и неудовольствия от их отсутствия, люди с выраженным фокусом продвижения более к ним чувствительны, а используемые ими стратегии достижения целей включают готовность достигать успеха или получать выигрыш. Система профилактики связана с переживанием удовольствия от отсутствия негативных исходов (потерь) и, соответственно, неудовольствия от их наличия, что порождает чувствительность к наличию или отсутствию негативного результата и использование бдительности с тем, чтобы избежать потерь. Например, как пишет Т. Хиггинс (Higgins et al., 1994), студент с выраженным фокусом продвижения, цель которого получить наивысший балл на экзамене, воспринимаемая им как достижение, может стремиться к этому путем чтения дополнительной литературы по теме или организации группы сокурсников для обсуждения вопросов курса. Студент с выраженным фокусом профилактики, который воспринимает цель получения наивысшего балла как ответственность, будет стараться минимизировать помехи, способные помешать ему достичь поставленной цели, стремясь убедиться, что он хорошо знает материал, или избежать какихлибо дополнительных раздражителей перед экзаменом. Эти стратегические различия в достижении желаемых состояний внутри двух мотивационных систем, согласно автору теории, могут быть рассмотрены в терминах теории обнаружения сигнала (Tanner, Swets, 1954). Тогда фокус профилактики будет пониматься как установка на уменьшение ошибок ложной тревоги (false alarm) и чувствительность к потерям, а фокус продвижения - как установка на увеличение попаданий (hit) и чувствительность к приобретениям (Higgins et al., 2001).

Фокус регуляции может быть как вызван ситуативно, например с помощью инструкций (Crowe, Higgins, 1997; Higgins, 1997), так и являться устоявшейся индивидуальной стратегией (так называемый доминирующий фокус - chronic regulatory focus), связанной с субъективной историей достижения успеха в различных видах деятельности. Развитие доминирующего фокуса регуляции авторы связывают, в частности, со стилями воспитания (Higgins, Silberman, 1998). Акцент родителей на позитивном результате, т.е. вознаграждение за желаемое поведение и отсутствие вознаграждения при отсутствии требуемого поведения, подкрепляет формирование фокуса продвижения. Акцент на негативном результате, т.е. наказание за несоответ- 
ствующее поведение и отсутствие наказания при соблюдении правил, подкрепляет формирование фокуса профилактики. На основании идеи о том, что доминирующий фокус регуляции формируется в течение жизни, Т. Хиггинс с соавт. разработали опросник по диагностике фокуса регуляции - Regulatory Focus Questionnaire (RFQ; Higgins et al., 2001).

За двадцатилетнюю историю своего существования теория фокуса регуляции приобрела достаточно большую известность и влиятельность. Начиная с первой публикации теории фокуса регуляции в 1997 г. количество ссылок на работы Т. Хиггинса, посвященные фокусу регуляции и опроснику, созданному для его диагностики, неуклонно растет (см. рисунок 2).

В рамках данной теории был не только предложен новый взгляд на мотивацию, связанную с достижением, но и обоснована связь репрезентации целей и стратегий их достижения с базовыми когнитивными механизмами (чувствительностью к определенным параметрам ситуации, вниманием, запоминанием и т.п.) (см., например: Molden, 2012).

Исследования, в которых регуляторный фокус измерялся с помощью опросника или же варьировался с помощью ситуативных факторов, показали различия в поведении, характерные для фокуса продвижения и профилактики. Выраженность фокуса продвижения положительно коррелировала со скоростью глобальной обработки информации и отрицательно - со скоростью локальной обработки, обратное было характерно для выраженности фокуса профилактики (Förster, Higgins, 2005). Также была обнаружена связь с балансом скорости/точности обработки информации: испытуемые с фокусом продвижения демонстрировали более быстрое, но менее точное выполнение

Рисунок 2

Количество цитирований (по данным Web of Science на июнь 2017 г.) ключевых статей Т. Хиггинса, посвященных изложению теории фокуса регуляции (Higgins, 1997, 1998) и опроснику по диагностике фокуса регуляции (Higgins et al., 2001)

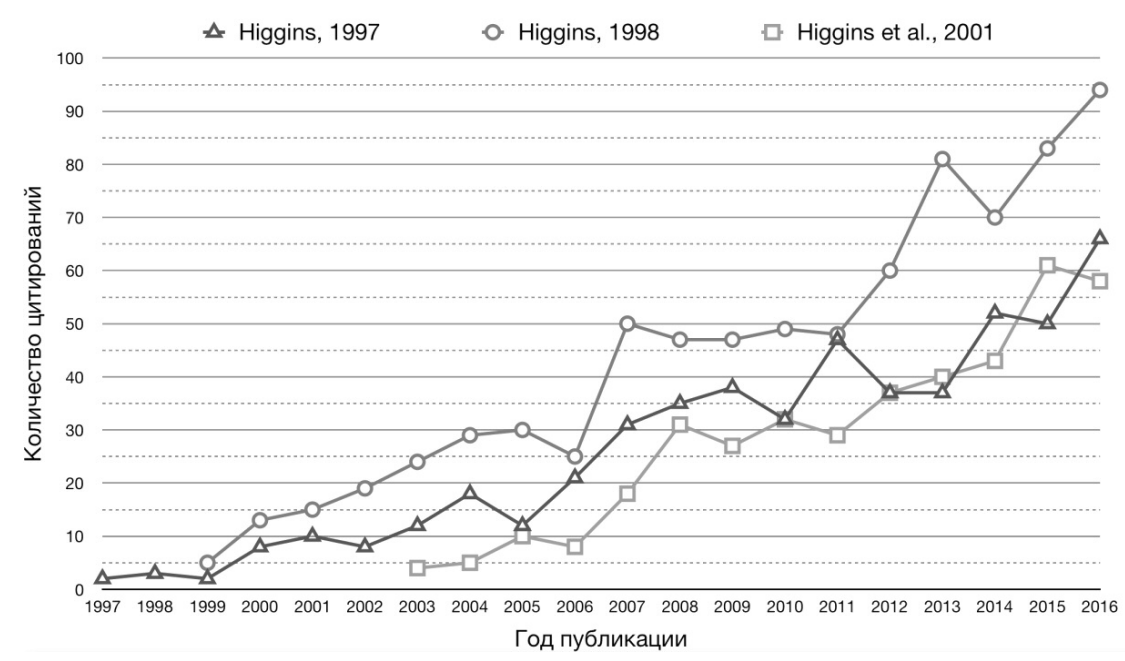


заданий, чем испытуемые с фокусом профилактики. Было показано, что по мере приближения к концу выполнения задачи у испытуемых с фокусом продвижения скорость увеличивалась, а точность падала, тогда как у испытуемых с фокусом профилактики - наоборот (Förster et al., 2003). Фокус регуляции оказался связан с решением творческих задач: индукция фокуса продвижения способствовала более быстрому нахождению решения в творческой задаче и генерации новых вариантов решений. Как предполагают авторы исследования, в основе таких различий лежат большая склонность к выбору рискованной стратегии ответа и активация поиска в памяти, связанного с новыми вариантами ответов (Crowe, Higgins, 1997; Friedman, Förster, 2001). Согласно точке зрения Хиггинса (Higgins, 1997), система регуляторного фокуса состоит из базовых механизмов, побуждающих к специфической настройке на обработку информации, аффективные переживания при этом дают дополнительную информацию касательно эффективности/неэффективности выбранного стиля обработки информации относительно достижения поставленной цели.

Обнаружена связь фокуса регуляции с особенностями взаимодействия в социальных ситуациях. Когда поведение людей основано на целях продвижения, они извлекают из социального окружения информацию, связанную с достижением успеха, т.е. они с большей вероятностью обратят внимание и будут вспоминать информацию, связанную с тем, как другие люди достигали успеха (Higgins, Tykocinski, 1992). Люди с таким фокусом регуляции хорошо настроены на эмоциональное состояние людей, связанное с успешным или неуспешным стремлением к выигрышу (т.е. к счастью или унынию соответственно) (Higgins et al., 1997). Более того, они демонстрируют высокую мотивацию и настойчивость в тех ситуациях, задачи в которых сформулированы в терминах продвижения (Shah et al., 1998). Когда же поведение людей регулируется целями профилактики, они обращают внимание на неудачи, вспоминают, как другие люди избегали неудач, и особо чувствительны к эмоциям других людей, связанных с их успешным или неуспешным избеганием негативных результатов (покой и тревожность соответственно). Они демонстрируют высокую мотивацию в тех ситуациях, задачи в которых сформулированы в терминах профилактики.

Таким образом, у людей с разным фокусом регуляции (продвижения или профилактики) обнаруживаются различные когнитивные и эмоциональные реакции, которые приводят к различному, а иногда даже противоположному поведению. В целом люди особо чувствительны к информации, которая соответствует их доминирующему регуляторному фокусу, они более мотивированы и результативны в ситуациях, когда поставленные задачи вынуждают их использовать те стратегии, которые соответствуют их фокусу (Higgins, 2000). Это наблюдение привело к разработке механизма регуляторного совпадения (regulatory fit). Постулируется, что если цель задания, с которым работает человек, совпадает с его доминирующим фокусом регуляции, то человек переживает регуляторное совпадение. Такое совпадение в целом приводит к более эффективному выполнению задания (Higgins, 2000, 2005), т.е. опосредованно, через регуляторное совпадение, фокус регуляции влияет на то, какова будет 
мотивация к выполнению задания и как будет оценен достигнутый результат (положительно или отрицательно) (Higgins, 2006).

Теория фокуса регуляции и механизм регуляторного совпадения активно исследуются в области социальной и организационной психологии, психологии рекламы и психологии спорта. Наибольший интерес исследователей вызывают эффекты регуляторного совпадения. Было показано, что соответствие доминирующего фокуса регуляции и фрейминга сообщений (акцент на приобретениях/акцент на потерях) приводит к большей эффективности последних. Так, например, если у человека преобладает фокус продвижения, он скорее обратит внимание на рекламу сока, если она сообщает о возможных приобретениях («Этот сок даст вам много энергии для новых свершений!»). Если у человека преобладает фокус профилактики, то его внимание больше привлечет сообщение о возможности предотвращения потерь (например: «Этот сок способствует профилактике болезней благодаря содержащимся в нем витаминам»). Применение данного эффекта исследуется на примере воздействия рекламных сообщений, в техниках переговоров, при разработке рекомендаций для врачей (что и как сообщать больному, чтобы он придерживался здоровьесберегающего поведения) и т.д. (Cesario et al., 2013; Mowle et al., 2014). Соответствие доминирующего фокуса регуляции и индуцированного задачей (например, с помощью инструкций тренера «Обязательно попади!» или «Только не промахнись!») способствует повышению эффективности выполнения когнитивных и двигательных задач (regulatory fit effects). Применение данного эффекта в последнее время все чаще исследуется у профессиональных спортсменов (футболистов, теннисистов, игроков в гольф и др.) (Kacperski, Kutzner, 2016; Kutzner et al., 2013; Plessner et al., 2009). При этом было показано, что несоответствие индивидуального фокуса регуляции и фрейма задачи, по-видимому, само по себе может вызывать напряжение и стресс (Schwab et al., 2015).

Все это свидетельствует об эвристичности теории фокуса регуляции. Еще одна область ее применения - кросс-культурные исследования мотивации. Согласно подходу Т. Хиггинса, культура играет важную роль в формировании фокусов регуляции. В теории предполагается, что восточноазиатские коллективистские культуры больше ориентированы на профилактику, тогда как западная индивидуалистская культура больше ориентирована на продвижение (Heine, Ruby, 2010; Higgins et al., 2008). Однако в реальных исследованиях обнаруживается более сложная картина (см., например: Kurman, Hui, 2011).

Интерес к теории мотивации Тори Хиггинса в России, несомненно, возрастает. В 2014 г. на русском языке была издана книга Х. Хэлворсона и Т. Хиггинса «Как глубинные установки влияют на наши желания и поступки» (Хэлворсон, Хиггинс, 2014), в которой излагаются базовые постулаты теории, был адаптирован на русский язык «опросник самостей» Т. Хиггинса (Кургинян, 2013), положение о регуляторном фокусе используется в когнитивно-психологических исследованиях (см., например: Агафонов, Козлов, 2014). Однако прямое сопоставление результатов отечественных исследований с работами иностранных коллег затруднено в связи с отсутствием методики диагностики фокуса регуляции, адаптированной для русскоязычной выборки. Мы 
обнаружили адаптации опросника на диагностику фокуса регуляции на финской (Leikas et al., 2009), китайской (Ip, Chiu, 2001), румынской (Rusu et al., 2015), польской (Bąk et al., 2015) выборках.

Цель настоящей работы - адаптация опросника «Regulatory Focus Questionnaire» на русскоязычной выборке. Адаптация опросника проводилась в несколько этапов. Ниже будут приведены результаты пилотажного и основного этапов исследования, в которое входили: проверка факторной структуры опросника, внутренней согласованности шкал, тест-ретестовой надежности; будет описано исследование, направленное на проверку валидности опросника.

\section{Структура оригинальной версии опросника}

Опросник на диагностику фокуса регуляции состоит из 11 вопросов и содержит две психометрически независимые шкалы. Шкала «Фокус продвижения» измеряет субъективное восприятие успеха, достигнутого посредством продвижения, и содержит 6 вопросов. Шкала «Фокус профилактики» измеряет субъективное восприятие успеха, достигнутого посредством профилактики, и содержит 5 вопросов. Вопросы в обеих шкалах сформулированы как в прямом, так и в обратном виде. Опросник основан на предположении, что люди с более высокими баллами по шкале «Фокус продвижения» будут использовать более рискованные стратегии при достижении цели, чем люди с меньшим количеством баллов по данной шкале. И независимо от этого люди с более высокими баллами по шкале «Фокус профилактики» будут более склонны к профилактике ошибок, чем люди с меньшим количеством баллов по данной шкале. Разработка опросника, проверка факторной структуры и надежности опросника проводились авторами на студенческой выборке (Higgins et al., 2001). Показана внутренняя согласованность пунктов шкалы: для шкалы «Фокус продвижения» - 0. 73, для шкалы «Фокус профилактики» - 0.8; корреляции между суммарными баллами по шкалам не обнаруживается. Отметим, что в теории фокуса регуляции предполагается, что как фокус продвижения, так и фокус профилактики отражают две разных формы реализации мотивации достижения. При проверке Т. Хиггинсом этого предположения с использованием шкалы мотивации достижения опросника Д. Джэксона (Jackson, 1974) была показана положительная корреляция выраженности каждого из фокусов с выраженностью мотивации достижения.

\section{Методика исследования и процедура адаптации}

В процедуре адаптации опросника приняли участие 536 человек, из них 158 - на пилотажном этапе, 308 - на основном этапе исследования, 70 человек в дополнительном исследовании, направленном на проверку валидности

\footnotetext{
${ }^{1}$ Авторы выражают благодарность своим коллегам и студентам И.И. Иванчею, И.В. Овчинниковой, М.И. Нелюбову, Р.В. Тихонову, Д.К. Урих., В.Д. Шеремет за помощь в сборе данных.
} 
опросника. В связи с тем что оригинальная версия опросника разрабатывалась на студенческой выборке, а известные нам адаптации опросников также ограничивались этим возрастным диапазоном, в выборку для русскоязычной адаптации включались испытуемые в возрасте от 17 до 23 лет.

Процесс адаптации методики осуществлялся в соответствии со стандартным методом адаптации зарубежных опросников (см., например: Бурлачук, 2006). Перевод методики с английского языка на русский был выполнен двумя независимыми экспертами: психологом, владеющим английским языком, и профессиональным переводчиком. В процессе перевода внимание уделялось сохранению содержания вопроса, а также сохранению грамматической формы высказывания.

После этого с целью проверки адекватности перевода был выполнен обратный перевод с русского на английский язык. Перевод осуществлялся профессиональным переводчиком, не знакомым с текстом оригинала. Полученный обратный перевод (с русского на английский) был прочитан Тори Хиггинсом (автором методики). Было получено подтверждение от автора оригинальной версии опросника сохранения содержания вопросов и инструкции с учетом двух этапов перевода опросника.

\section{Пилотажное исследование}

На первом этапе было проведено пилотажное исследование, целями которого были: проверка факторной валидности методики; проверка надежности шкал методики по внутренней согласованности их пунктов; проверка понимания испытуемыми вопросов методики. В пилотажном исследовании приняли участие 158 человек, в возрасте от 17 до 23 лет $(\mathrm{M}=20.5$ года, $\sigma=1.7) ; 122$ женщины, 36 мужчин. Все опросы проводились в индивидуальном порядке на добровольной основе.

В результате применения конфирматорного факторного анализа (КФА) была подтверждена двухфакторная ортогональная структура опросника, правда, с потерей одного пункта (№ 9). Для проверки внутренней согласованности рассчитывался коэффициент $\alpha$ Кронбаха. Были получены следующие показатели: шкала «Фокус продвижения» $-\alpha$ Кронбаха $=0.523$, шкала «Фокус профилактики» - $\alpha$ Кронбаха $=0.677$. Корреляции между суммарными показателями шкал обнаружено не было $(r=0.114, p=0.152)$.

Мы предположили, что сохранение факторной структуры при невысокой внутренней согласованности может быть связано с не очень понятными формулировками вопросов. Для основного этапа нами было сделано несколько вариантов формулировок каждого вопроса с тем, чтобы подобрать формы высказывания, наиболее адекватные нормам русского языка. В работе над формулировками приняли участие два эксперта-филолога. При коррекции формулировок содержание вопроса практически не изменялось (например, для вопроса англоязычной версии «Do you often do well at different things that you try?» были сформулированы следующие варианты перевода: вариант 1. Как часто вы преуспеваете в ваших начинаниях?; вариант 2. Как часто вам 
удается достичь успеха в новых видах деятельности?). В некоторых случаях для избегания формулировки вопроса с двойным отрицанием предлагался вариант перевода, ведущий к изменению подсчета баллов по шкале (например, для вопроса англоязычной версии «Compared to most people, are you typically unable to get what you want out of life?» были предложены следующие варианты: вариант 1. В сравнении с другими людьми как часто вы не получаете от жизни то, чего хотите?; вариант 2 (обратная формулировка вопроса): Обычно я добиваюсь того, чего хочу. Для каждого вопроса было сформулировано 2-3 разных варианта перевода, что в итоге составило 28 вопросов. В связи с небольшим количеством вопросов было принято решение предъявлять испытуемым все 28 вопросов, так как мы предполагали, что влияние последовательности вопросов будет незначительным.

\section{Основной этап}

В основном этапе адаптации опросника приняли участие 308 человек, в возрасте от 17 до 23 лет ( $\mathrm{M}=20.8$ года, $\sigma=1.76) ; 207$ женщин, 101 мужчина. Все опрошенные - студенты различных вузов.

Испытуемые заполняли онлайн-версию опросника. При описании оригинальной версии опросника мы не нашли указаний на предпочтительные формы его заполнения, однако в описанных выше исследованиях, включающих этот опросник, используются как онлайн-, так и офлайн-версии. Вопросы предъявлялись по одному и были разбиты на условные блоки: сначала первые формулировки всех 11 вопросов, потом вторые варианты формулировок и затем - третьи варианты для нескольких вопросов.

\section{Анализ надежности русскоязычной версии}

Для исследования надежности был проведен анализ внутренней согласованности шкал теста. На первом этапе мы выбрали 11 из 28 формулировок вопросов, которые показали наилучшую внутреннюю согласованность по каждой из шкал. Для проверки внутренней согласованности рассчитывался коэффициент $\alpha$ Кронбаха (Наследов, 2013; Шмелев, 2013) и GLB (Guttman's L2) (Bentler, 1972; Shapiro, ten Berge, 2000). Были получены следующие показатели надежности шкал по внутренней согласованности их пунктов: шкала «Фокус продвижения» (6 вопросов) - 0.770 (GLB (Guttman’s L2) = 0.777); шкала «Фокус профилактики» (5 вопросов) - 0.784 (GLB (Guttman’s L2) = 0.794).

Удаление любого из оставшихся пунктов не приводило к увеличению надежности шкал, поэтому по критерию надежности данный набор из 11 оставшихся вопросов является оптимальным для двух шкал методики.

Для проверки факторной валидности двухшкального опросника применялся конфирматорный факторный анализ (КФА) в отношении отобранных 11 вопросов (программа SPSS AMOS). Допустимость применения КФА проверяется по достаточному объему выборки и по многомерной нормальности переменных. Соотношение объема выборки $(\mathrm{N}=308)$ и числа оцениваемых 
параметров модели $(\mathrm{T}=28)$ существенно превышает недопустимо низкий порог 5, следовательно, объем выборки достаточен. Многомерная нормальность проверяется по критическому отношению (C.R.) для многомерного эксцесса, который не должен заметно превышать 5 (Kline, 2011). В нашем случае C.R. = 7.211, что указывает на отклонение распределения переменных от многомерной нормальности. В этом случае, однако, возможно применение метода анализа, приблизительно свободного от распределения (ADF, Asimptotically Distribution Free) (Наследов, 2013; Byrne, 2010). Этот метод, доступный в программе AMOS, и был нами применен для КФА.

В качестве исходной гипотетической (априорной) модели использовалась модель оригинальной версии методики. Финальная модель (блок-схема) представлена на рисунке 3. Степень соответствия модели исходным данным оценивалась по следующим индексам согласия (приведены под блок-схемой на рисунке 3): отношение Хи-квадрат к числу степеней свободы $(\mathrm{CMIN} / \mathrm{df})<2.0$, $p$-уровень для CMIN $(p)>0.05$, индекс согласия Goodness of Fit Index (GFI) > 0.95, индекс согласия Comparative Fit Indices (CFI) > 0.95, среднеквадратичная ошибка аппроксимации Root Mean Square Error of Approximation (RMSEA) < 0.05, с eе

\section{Финальная модель двухшкальной методики}

Рисунок 3

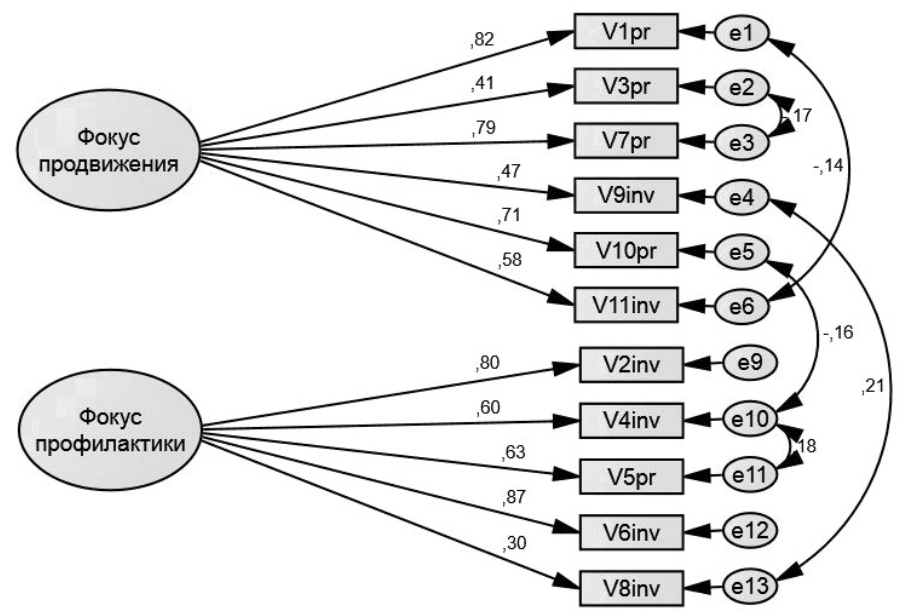

CMIN=52,580; df=39; p=,072; GFI=,971; CFI=,957; RMSEA=,034; Pclose=,884

Примечание. В прямоугольных контурах - явные переменные-индикаторы, в округлых контурах - латентные Факторы (шкалы), и «ошибки» измерения; числа у направленных стрелок стандартизованные коэффициенты регрессии, числа у ненаправленных стрелок - величины корреляций между переменными. Номера вопросов (V1-V11) соответствуют порядку вопросов, приведенных в Приложении 1. Код «рг» означает, что по данному пункту шкалы производится прямой подсчет баллов, код «inv» означает, что по данному пункту шкалы производится обратный подсчет баллов (см. ключ к методике в Приложении 1). 
близостью согласия (Pclose) > 0.50 (Byrne, 2010, p. 73-84). Пронумерованный список вопросов, представленный на блок-схеме, приведен в приложении 1. Все индексы согласия, приведенные под блок-схемой, свидетельствуют об отличном соответствии модели исходным данным. Все регрессионные коэффициенты модели статистически достоверны $(p<0.01)$. Дисперсии латентных переменных (факторов) статистически достоверно отличаются от $0(p<0.001)$. Корреляция между факторами статистически не достоверно отличается от нуля $(p>0.1)$. Таким образом, каждый пункт методики является индикатором только одной шкалы (отсутствие «стрелок» на блок-схеме означает, что соответствующая связь пренебрежимо мала). Факторы (шкалы) не коррелируют (ортогональны). Статистическая значимость дисперсий факторов (шкал) означает, что эти шкалы достаточно хорошо дифференцируют испытуемых. Таким образом, факторная валидность двухшкального опросника, состоящего из 11 пунктов, полностью подтверждается.

Результаты анализа показали соответствие структуры адаптируемой методики двухфакторной ортогональной структуре оригинальной версии опросника.

Полученные показатели внутренней согласованности соответствуют данным, полученным при разработке оригинальной англоязычной версии опросника ( $\alpha$ Кронбаха для шкалы «Фокус продвижения» 0.73 и для шкалы «Фокус профилактики» - 0.80) (Higgins et al., 2001).

Описательные статистики по каждой шкале приведены в таблице 1.

В описании оригинальной версии опросника мы не обнаружили указаний на то, различаются ли средние значения по шкалам у мужчин и женщин (нет данных, обосновывающих как возможное наличие влияния фактора пола, так и его отсутствие). Мы решили проконтролировать этот показатель на нашей выборке, чтобы уточнить возможное наличие половых различий. Мы сравнили средние суммарные баллы по шкалам «Фокус профилактики» и «Фокус продвижения» для мужчин и женщин, вошедших в выборку для адаптации. Влияние фактора пола обнаружено не было (данные см. в таблице 2). Наши данные свидетельствуют о том, что исследовательский вариант опросника можно использовать как на мужской, так и на женской выборке.

В выборку для проведения ретеста вошло 96 человек (68 женщин, 28 мужчин). Повторный опрос проводился спустя 4 недели после первичного опроса. Были подсчитаны корреляция между суммарными баллами по каждой из шкал, а также различия средних значений. Полученные данные приведены в таблице 3.

Таблица 1

Описательные статистики, полученные по каждой из шкал опросника

\begin{tabular}{|l|c|c|c|c|c|}
\hline \multicolumn{1}{|c|}{ Шкалы опросника } & M & SD & Min & Max & Асимметрия/эксцесс \\
\hline $\begin{array}{l}\text { Шкала «Фокус продвижения» } \\
\text { (6 вопросов) }\end{array}$ & 21.3 & 4.29 & 8 & 30 & $-0.390 / 0.076$ \\
\hline $\begin{array}{l}\text { Шкала «Фокус профилактики» } \\
\text { (5 вопросов) }\end{array}$ & 16.6 & 3.79 & 5 & 24 & $-0.526 /-0.125$ \\
\hline
\end{tabular}


Таблица 2

Данные по шкалам опросника, полученные на мужской и женской подвыборках

\begin{tabular}{|c|c|c|c|c|c|c|}
\hline & Пол & $\mathbf{N}$ & $\mathbf{M}$ & SD & $\begin{array}{c}\text { t-критерий, } \\
\text { р-уровень }\end{array}$ & $\begin{array}{c}\text { Критерий ра- } \\
\text { венства дисперсий }\end{array}$ \\
\hline \multirow{2}{*}{$\begin{array}{l}\text { Фокус } \\
\text { продвижения }\end{array}$} & женский & 207 & 21.4 & 4.18 & \multirow{2}{*}{$\begin{array}{l}t=0.349 \\
p=0.727\end{array}$} & \multirow{2}{*}{$\begin{array}{l}F=0.444 \\
p=0.506\end{array}$} \\
\hline & мужской & 101 & 21.2 & 4.52 & & \\
\hline \multirow{2}{*}{$\begin{array}{l}\text { Фокус } \\
\text { профилактики }\end{array}$} & женский & 207 & 16.7 & 3.77 & \multirow{2}{*}{$\begin{array}{l}t=0.930 \\
p=0.353\end{array}$} & \multirow{2}{*}{$\begin{array}{l}F=0.122 \\
p=0.727\end{array}$} \\
\hline & мужской & 101 & 16.3 & 3.83 & & \\
\hline
\end{tabular}

Таблииа 3

Результаты проверки ретестовой надежности опросника

\begin{tabular}{|l|c|c|c|c|}
\hline & $\begin{array}{c}\text { Корреляция } \\
\text { суммарных бал- } \\
\text { лов по шкале } \\
\text { (тест-ретест) }\end{array}$ & $\begin{array}{c}\text { Средний суммар- } \\
\text { ный показатель } \\
\text { шкалы/станд. } \\
\text { откл. - тест }\end{array}$ & $\begin{array}{c}\text { Средний суммар- } \\
\text { ный показатель } \\
\text { шкалы/станд. } \\
\text { откл. - ретест }\end{array}$ & $\begin{array}{c}\boldsymbol{t} \text {-критерий, } \\
\boldsymbol{p} \text {-уровень }\end{array}$ \\
\hline $\begin{array}{l}\text { Фокус продви- } \\
\text { жения }\end{array}$ & $0.872^{* *}$ & $21.3 / 4.29$ & $20.9 / 4.1$ & $\begin{array}{c}t=-0.559 \\
p=0.577\end{array}$ \\
\hline $\begin{array}{l}\text { Фокус профи- } \\
\text { лактики }\end{array}$ & $0.879 *$ & $16.6 / 3.79$ & $16.9 / 3.7$ & $\begin{array}{c}t=-1.045 \\
p=0.299\end{array}$ \\
\hline
\end{tabular}

Данные ретестовой выборки были дополнительно подвергнуты КФА. В результате была получена финальная модель, в точности соответствующая приведенной на рисунке 3 , индексы согласия: $\mathrm{CMIN}=46.059 ; \mathrm{df}=40 ; p=0.236$; GFI $=0.962 ; \mathrm{CFI}=0.984 ;$ RMSEA $=0.040 ;$ Pclose $=0.600$. Несмотря на недостаточный объем выборки $(\mathrm{N}=96 ; \mathrm{T}=26)$, индексы согласия демонстрируют очень хорошее соответствие модели исходным данным, а ее идентичность финальной модели (рисунок 3) подтверждает факторную валидность адаптируемой методики.

Отобранные в результате основного этапа адаптации 11 вопросов были еще раз подвергнуты обратному переводу на английский язык и повторно сверены с автором методики. Было получено подтверждение сохранения логики опросника и содержания вопросов.

\section{Проверка валидности опросника}

В теории Т. Хиггинса утверждается, что особенности доминирующего фокуса регуляции должны проявляться в различных стратегиях при принятии решений. Люди, у которых преобладает фокус продвижения, будут склонны использовать более рискованные стратегии, делая акцент на вероятности приобретения, тогда как люди с фокусом профилактики будут использовать более осторожные стратегии, оценивая вероятность потерь. Следует ожидать 
(Higgins et al., 2001), что фокус регуляции может оказывать влияние на подверженность классическим когнитивным искажениям при принятии решений (Kahneman, Tversky, 1979).

В исследовании Хиггинса с соавт. (Higgins et al., 2001, исследования 1a, 1b) была показана связь типа ошибок при решении задач и выраженности соответствующего фокуса регуляции. Для проверки валидности опросника, адаптированного на русскоязычной выборке, мы решили воспроизвести это исследование. Опишем его подробнее.

В исследовании использовались две задачи. Первая задача провоцировала выбор между двумя путешествиями (невозвратные вклады), запланированными на одно и то же время. При этом стоимость одного из них в два раза меньше, чем другого, но менее ценное при этом доставит больше удовольствия. Таким образом, необходимо выбрать либо избегание бо́льших финансовых потерь, либо получение большего удовольствия. В рамках исследования предполагалось, что испытуемые с более выраженным фокусом продвижения вследствие их общей нацеленности на приобретение будут реже отказываться от выбора, связанного с бо́льшим удовольствием.

В первом исследовании испытуемым давалась задача, взятая из исследования Х. Аркса и К. Блумер (Arkes, Blumer, 1985; ниже задача приведена в нашем переводе):

Представьте себе, что вы потратили 100 долларов на билет на поездку в Мичиган на выходные. Несколько недель спустя вы покупаете билет на поездку на выходные дни в Висконсин за 50 долларов. Вам кажется, что поездка в Висконсин понравится вам больше, чем поездка в Мичиган. И только вы собираетесь положить только что купленный билет в Висконсин в кошелек, как замечаете, что и поездка в Мичиган, и поездка в Висконсин приходятся на одни и те же выходные дни! Уже слишком поздно продать какой-либо из билетов, и ни один из них нельзя вернуть. Вы должны использовать только какой-то один из билетов. В какую поездку вы поедете?

Авторы полагали, что испытуемые с более выраженным фокусом продвижения будут с большей вероятностью выбирать поездку в Висконсин, а связи с выраженностью фокуса профилактики обнаружено не будет. По результатам исследования (см. 1a в: Higgins et al., 2001), несмотря на то что 80\% испытуемых выбирали поездку в Висконсин, была также обнаружена положительная связь между выбором поездки в Висконсин и выраженностью фокуса продвижения, связи с выраженностью фокуса профилактики обнаружено не было.

Во второй задаче (Higgins et al., 2001, исследование 1b) выбор стоял между вкладом дополнительных ресурсов в окончание проекта (который с высокой вероятностью будет неуспешным) и полным отказом от участия в нем. Так как люди с выраженным фокусом профилактики более чувствительны к возможным потерям, они будут менее склонны вкладывать дополнительные ресурсы в убыточный проект. Для проверки этого предположения авторы взяли задачу из исследования Х. Аркса и К. Блумер (Arkes, Blumer, 1985, эксперимент 3; задача приведена в нашем переводе): 
Являясь президентом авиакомпании, вы инвестировали 10 миллионов долларов денег компании в исследовательский проект. Цель проекта - построить самолет, который не смогут замечать обычные радары. Когда ваш проект уже на 90\% завершен, другая компания представляет самолет, который также не могут замечать радары. Причем их самолет быстрее и его строительство обходится компании дешевле, чем ваш проект. Вопрос: станете ли вы инвестировать оставшиеся $10 \%$ денег в окончание своего проекта?

В исследовании авторов 70\% испытуемых ответили «да», однако была показана связь между выраженностью фокуса профилактики и тенденцией отвечать «нет».

Для проверки валидности русскоязычной версии опросника было проведено два исследования.

В первом исследовании приняли участие 276 человек, входивших в исходную выборку для адаптации опросника. После заполнения опросника испытуемым предлагалось решить первую задачу. После консультации с экспертами лингвистами и психологами была предложена следующая формулировка задачи 1:

Представьте, что вы потратили 6000 рублей на билеты на выходные в Москву. Через несколько недель вы купили билеть на выходнье в Казань за 3000 рублей. Причем поехать в Казань вы хотите больше, чем в Москву. Просматривая вечером билеты, вы понимаете, что они куплены на одни и те же выходные! Возможности купить новые билеты у вас уже нет, возвращать купленные тоже слишком поздно. Вам придется поехать лишь в один из этих городов. Куда вы поедете?

Мы ожидали, что испытуемые с более выраженным фокусом продвижения будут чаще предпочитать поездку в Казань, чем испытуемые с менее выраженным фокусом продвижения.

Мы обнаружили, что в этой задаче $82 \%$ испытуемых выбирают поездку в Казань (что соответствует данным, полученным исследователями по аналогичной задаче, сформулированной на английском языке). Далее были рассчитаны суммарные показатели по шкалам «Фокус продвижения» и «Фокус профилактики», по каждой из шкал выборка была поделена по среднему - соответственно на более и менее выраженный показатель по выборке. Связь между выраженностью фокусов продвижения и профилактики и ответом на задачу была проанализирована с помощью таблиц сопряженности (см. таблицу 4).

Таблица 4

Ответы на задачу 1 в связи с выраженностью фокусов продвижения и профилактики

\begin{tabular}{|l|l|c|c|}
\hline \multirow{2}{*}{$\begin{array}{c}\text { Выраженность фокусов регуляции (ыыше/ниже } \\
\text { среднего по выборке) }\end{array}$} & \multicolumn{1}{c|}{ Ответ на задачу } \\
\cline { 2 - 4 } & Москва & Казань \\
\hline \multirow{2}{*}{$\begin{array}{l}\text { Выраженность фокуса } \\
\text { продвижения }\end{array}$} & низкая $(\mathrm{M}<21.3)$ & 32 чел. $(23.2 \%)$ & 106 чел. (76.8\%) \\
\cline { 2 - 4 } & высокая $(\mathrm{M}>21.3)$ & 17 чел. (12.3\%) & 121 чел. (87.7\%) \\
\hline \multirow{2}{*}{$\begin{array}{l}\text { Выраженность фокуса } \\
\text { профилактики }\end{array}$} & низкая $(\mathrm{M}<16.6)$ & 19 чел. (15.8\%) & 101 чел. (84.2\%) \\
\cline { 2 - 4 } & высокая $(\mathrm{M}>16.6)$ & 30 чел. (19.2\%) & 126 чел. (80.8\%) \\
\hline
\end{tabular}


Полученные результаты позволили подтвердить выдвинутую гипотезу: более выраженный фокус продвижения связан с более частым предпочтением поездки в Казань, чем менее выраженный фокус продвижения (точный критерий Фишера, $p=0.027)$. Связи выраженности фокуса профилактики с предпочтением того или иного ответа на задачу не обнаружено (точный критерий Фишера, $p=0.526)$.

Во втором исследовании приняли участие 70 человек (в возрасте от 18 до 22 лет, $\mathrm{M}=20.2, \sigma=1.24 ; 49$ женщин, 21 мужчина), не входившие в исходную выборку для адаптации опросника. Испытуемые для этого исследования набирались отдельно от основного этапа адаптации. Испытуемые заполняли опросник в окончательной редакции (только ранее отобранные 11 вопросов), по окончании заполнения опросника решали предложенную задачу. После консультации с экспертами лингвистами и психологами была предложена следующая формулировка задачи 2:

Представьте, что вы руководите крупной авиакомпанией и уже потратили 1 миллиард рублей из денег компании на исследовательский проект. Цель проекта - построить самолет, который не смогут замечать обычные радары. Когда ваш проект уже на 90\% завершен, другая компания представляет самолет, который также не могут замечать радары. Причем их самолет быстрее, и его строительство обходится компании дешевле. Вопрос: станете ли вы инвестировать оставшиеся 10\% денег в создание своего самолета?

Для начала мы проверили внутреннюю согласованность шкал опросника. Это было сделано для независимой проверки ранее выделенных 11 вопросов.

Показатель $\alpha$ Кронбаха для фокуса продвижения $=0.758$, показатель $\alpha$ Кронбаха для фокуса профилактики $=0.789$. Корреляции между суммарными показателями шкал не обнаружено $(r=-0.078, p=0.522)$.

Мы обнаружили, что в этой задаче $65.7 \%$ испытуемых выбирают ответ «да». Далее были рассчитаны суммарные показатели по шкалам «Фокус продвижения» и «Фокус профилактики», по каждой из шкал выборка была поделена по среднему - соответственно на более и менее выраженный показатель по выборке. Связь между выраженностью фокусов продвижения и профилактики и ответом на задачу была проанализирована с помощью таблиц сопряженности (см. таблицу 5).

Таблица 5

Ответы на задачу 2 в связи с выраженностью фокусов продвижения и профилактики

\begin{tabular}{|l|l|c|c|}
\hline \multirow{2}{*}{$\begin{array}{c}\text { Выраженность фокусов регуляции (выше/ниже } \\
\text { среднего по выборке) }\end{array}$} & \multicolumn{2}{c|}{ Ответ на задачу } \\
\cline { 2 - 4 } & да & нет \\
\hline \multirow{2}{*}{$\begin{array}{l}\text { Выраженность фокуса } \\
\text { продвижения }\end{array}$} & низкая $(\mathrm{M}<20.9)$ & 21 чел. $(75.0 \%)$ & 7 чел. $(25.0 \%)$ \\
\cline { 2 - 4 } & высокая $(\mathrm{M}>20.9)$ & 25 чел. $(59.5 \%)$ & 17 чел. $(40.5 \%)$ \\
\hline $\begin{array}{l}\text { Выраженность фокуса } \\
\text { профилактики }\end{array}$ & низкая $(\mathrm{M}<17.2)$ & 22 чел .(78.6\%) & 6 чел. $(21.4 \%)$ \\
\cline { 2 - 4 } & высокая $(\mathrm{M}>17.2)$ & 24 чел. $(57.1 \%)$ & 18 чел. $(42.9 \%)$ \\
\hline
\end{tabular}


Статистически достоверной связи между выраженностью фокуса продвижения и выбором одного из вариантов ответа на задачу не обнаружено (точный критерий Фишера, $p=0.208)$. Связь выраженности фокуса профилактики с выбором ответа «нет» не достигает уровня статистической значимости (точный критерий Фишера, $p=0.077)$. Полученные результаты не позволили однозначно подтвердить выдвинутую гипотезу. Однако направленность полученных различий в целом соответствует предсказаниям.

Отметим, что проверка валидности русскоязычной версии опросника проводилась через воспроизведение результатов исследования Т. Хиггинса с coавт. (Higgins et al., 2001), в котором была показана связь выраженности того или иного фокуса регуляции и стратегий принятия решений. Полученные данные в целом свидетельствуют о том, что русскоязычная версия опросника, позволяет предсказывать такое же направление связи, как и в оригинальном исследовании авторов. Наши данные не позволяют говорить о том, что можно проводить индивидуальную диагностику склонности к когнитивным искажениям. Скорее, мы можем констатировать, что в целом по выборке наблюдается общая тенденция, в соответствии с которой особенности доминирующего фокуса регуляции проявляются в различных стратегиях принятия решений в ситуации неопределенности.

\section{Общая характеристика итоговой версии опросника, адаптированной для русскоязычной выборки}

Предполагаемые адресаты опросника - юноши и девушки в возрасте от 17 до 23 лет.

Представление опросника и обработка результатов. Методика Диагностика фокуса регуляции состоит из двух шкал. Шкала диагностики фокуса продвижения включает 6 вопросов, диагностики фокуса профилактики -5 вопросов. Утверждения отдельных шкал перемешаны. Испытуемые дают ответы, используя шкалу от 1 (никогда/совершенно не согласен) до 5 (очень часто/совершенно согласен). При подсчете баллов ответы на «обратные» утверждения меняются на противоположные значения. (Опросник и ключ к опроснику см. в приложении 1.) Общий показатель по каждой шкале вычисляется путем сложения баллов.

\section{Заключение}

Результаты применения КФА показали сохранение двухфакторной ортогональной структуры опросника, шкалы методики обладают достаточно высокой надежностью по внутренней согласованности их пунктов, по ретестовой надежности. Количество вопросов, вошедших в русскоязычную версию, соответствует оригинальной версии опросника. В целом подтверждается и конструктная валидность шкал, однако, на наш взгляд, их дальнейшая проверка с помощью других психологических методик представляет несомненный интеpec. 
Дальнейшая работа по проверке и обоснованию конструктной валидности адаптированной методики может быть направлена на проверку связей с такими релевантными с точки зрения теории Т. Хиггинса переменными, как стратегия принятия решения в ситуации неопределенности и склонность к риску (Higgins, 1997, 1998). Также важной представляется проверка положения о связи высокой выраженности обоих фокусов регуляции с высокими показателями мотивации достижения (см.: Molden et al., 2008) на русскоязычной выборке. Это позволит прояснить структуру связей между такими теоретическими конструктами, как «мотивация достижения успеха/избегания неудач» и «фокус регуляции (продвижение/профилактика)», и обосновать дальнейшие перспективы применения методики диагностики фокуса регуляции в практических целях.

\section{Литература}

Агафонов, А. Ю., Козлов, Д. Д. (2014). Познавательные стратегии в работе сознания и бессознательного. Известия Самарского научного центра РАН, 16(2), 864-872.

Бурлачук, Л. Ф. (2006). Психодиагностика: учебник для вузов. СПб.: Питер.

Кургинян, С. С. (2013). Апробация опросника самостей. Психология. Журнал Высшей школь экономики, 10(2), 122-137.

Наследов, А. Д. (2013). IBM SPSS 20 и AMOS: профессиональный статистический анализ данных. СПб.: Питер.

Хэлворсон, Х., Хиггинс, Т. (2014). Психология мотиващии. Как глубинные установки влияют на наши желания и поступки. М.: Манн, Иванов, Фербер.

Шмелев, А. Г. (2013). Практическая тестология. Тестирование в образовании, прикладной психологии и управлении персоналом. М.: ООО «ИПЦ «Маска».

Ссылки на зарубежные источники см. в разделе References после англоязычного блока.

\section{Инструкция и текст опросника}

Приложение 1

\section{ИНСТРУКЦИЯ}

Вопросы ниже касаются того, КАК ЧАСТО определенные события происходят или происходили в вашей жизни.

Пожалуйста, укажите ваш ответ, обведя соответствующую цифру после вопроса. Воспользуйтесь шкалой, приведенной после каждого вопроса. Неподписанные цифры соответствуют промежуточным вариантам ответов между подписанными.

\begin{tabular}{|c|c|c|c|c|c|}
\hline \multirow[b]{2}{*}{ Обычно я добиваюсь того, чего хочу } & 1 & 2 & 3 & 4 & 5 \\
\hline & $\begin{array}{c}\text { Никогда или } \\
\text { очень редко }\end{array}$ & & & & Очень часто \\
\hline \multirow{2}{*}{$\begin{array}{l}\text { Переходили ли вы в детстве границы доз- } \\
\text { воленного, делая то, что ваши родители } \\
\text { вам запрещали? }\end{array}$} & 1 & 2 & 3 & 4 & 5 \\
\hline & $\begin{array}{c}\text { Никогда или } \\
\text { очень редко }\end{array}$ & & & & Очень часто \\
\hline
\end{tabular}




\begin{tabular}{|c|c|c|c|c|c|}
\hline \multirow{2}{*}{$\begin{array}{l}\text { Как часто завершение какого-либо дела } \\
\text { вдохновляло вас на дальнейшее продол- } \\
\text { жение работы в этом направлении? }\end{array}$} & 1 & 2 & 3 & 4 & 5 \\
\hline & $\begin{array}{l}\text { Никогда или } \\
\text { очень редко }\end{array}$ & & & & Очень часто \\
\hline \multirow[b]{2}{*}{$\begin{array}{l}\text { Как часто вы «играли на родительских } \\
\text { нервах», когда были ребенком? }\end{array}$} & 1 & 2 & 3 & 4 & 5 \\
\hline & $\begin{array}{c}\text { Никогда или } \\
\text { очень редко }\end{array}$ & & & & Очень часто \\
\hline \multirow[b]{2}{*}{ Слушались ли вы ваших родителей? } & 1 & 2 & 3 & 4 & 5 \\
\hline & $\begin{array}{l}\text { Никогда или } \\
\text { очень редко }\end{array}$ & & & & Очень часто \\
\hline \multirow{2}{*}{$\begin{array}{l}\text { Как часто в детстве вы совершали поступ- } \\
\text { ки, которые ваши родители явно не одоб- } \\
\text { ряли? }\end{array}$} & 1 & 2 & 3 & 4 & 5 \\
\hline & $\begin{array}{c}\text { Никогда или } \\
\text { очень редко }\end{array}$ & & & & Очень часто \\
\hline \multirow[b]{2}{*}{$\begin{array}{l}\text { Как часто вы преуспеваете в ваших начи- } \\
\text { наниях? }\end{array}$} & 1 & 2 & 3 & 4 & 5 \\
\hline & $\begin{array}{l}\text { Никогда или } \\
\text { очень редко }\end{array}$ & & & & Очень часто \\
\hline \multirow[b]{2}{*}{ Я бываю неосторожен } & 1 & 2 & 3 & 4 & 5 \\
\hline & $\begin{array}{l}\text { Никогда или } \\
\text { очень редко }\end{array}$ & & & & Очень часто \\
\hline \multirow{2}{*}{$\begin{array}{l}\text { Как часто при решении важной для вас } \\
\text { задачи вам кажется, что вы справляетесь } \\
\text { хуже, чем хотели бы? }\end{array}$} & 1 & 2 & 3 & 4 & 5 \\
\hline & $\begin{array}{l}\text { Никогда или } \\
\text { очень редко }\end{array}$ & & & & Очень часто \\
\hline \multirow{2}{*}{$\begin{array}{l}\text { Я чувствую, что двигаюсь к достижению } \\
\text { успеха в своей жизни }\end{array}$} & 1 & 2 & 3 & 4 & 5 \\
\hline & $\begin{array}{c}\text { Совершенно } \\
\text { не согласен }\end{array}$ & & & & $\begin{array}{c}\text { Совершенно } \\
\text { согласен }\end{array}$ \\
\hline \multirow{2}{*}{$\begin{array}{l}\text { В моей жизни мало хобби и увлечений, } \\
\text { отвечающих моим интересам, заниматься } \\
\text { которыми мне действительно хочется }\end{array}$} & 1 & 2 & 3 & 4 & 5 \\
\hline & $\begin{array}{l}\text { Совершенно } \\
\text { не согласен }\end{array}$ & & & & $\begin{array}{c}\text { Совершенно } \\
\text { согласен }\end{array}$ \\
\hline
\end{tabular}

Ключ. По каждой шкале подсчитывается сырой балл по следующим формулам:

Фокус продвижения: № $1+$ № 3 + № $7+(6-$ №9) + № $10+(6$ - № 11).

Фокус профилактики: (6 - № 2) + (6 - № 4) + № 5 + (6 - № 6) + (6 - №8).

Гершкович Валерия Александровна - доцент, кафедра проблем конвергенции естественных и гуманитарных наук, Санкт-Петербургский государственный университет, кандидат психологических наук.

Сфера научных интересов: когнитивная психология, когнитивный контроль, научение, соревновательный стресс, мотивация достижения, иллюзии памяти.

Контакты: valeria.gershkovich@gmail.com 
Морошкина Надежда Владимировна - старший научный сотрудник, лаборатория когнитивных исследований, Санкт-Петербургский государственный университет, кандидат психологических наук.

Сфера научных интересов: когнитивная психология, имплицитное научение и память, сознание и когнитивное бессознательное, эффекты фрейма, мотивация достижения.

Контакты: moroshkina.n@gmail.com

Кулиева Алмара Кудрат кызы - аспирант, кафедра общей психологии, СанктПетербургский государственный университет.

Сфера научных интересов: когнитивная психология, психология сознания, психология воли, зрительные иллюзии.

Контакты: almara.kulieva@gmail.com

Наследов Андрей Дмитриевич - доцент, факультет психологии, Санкт-Петербургский государственный университет, кандидат психологических наук.

Сфера научных интересов: применение математических методов и моделей в психологии; методы измерений в психологии; разработка методик психодиагностики.

Контакты: andrey.nasledov@gmail.com

\title{
Measure of Chronic Regulatory Focus: Adaptation of T. Higgins Regulatory Focus Questionnaire in Russia
}

\author{
V.A. Gershkovicha ${ }^{\mathrm{a}}$ N.V. Moroshkina ${ }^{\mathrm{a}}$, A.K. Kulievaa ${ }^{\mathrm{a}}$, A.D. Nasledov ${ }^{\mathrm{a}}$ \\ a Saint Petersburg State University, 7/9 Universitetskaya emb., Saint Petersburg, 199034, Russian \\ Federation
}

\begin{abstract}
The adaptation of Regulatory Focus Questionnaire, RFQ for Russian sample is described. According to regulatory focus theory the goal-directed behavior is regulated by two different motivational systems (self-regulation strategic systems) - promotion focus and prevention focus. People with promotion focus strive for positive end-states, gains, use eager means; people with prevention focus strive for security and non-losses and use vigilant means. After back-translating procedure, the factor structure (confirmatory factor analysis), internal reliability and testretest reliability were explored. Construct validity was explored in the studies of interconnection of chronic regulatory focus and cognitive biases in decision making. The studies were aimed to replicate on a Russian sample the study by Higgins et al., 2001. Overall 536 people (aged 17 to 23) participated in the study (158 in the pilot study, 308 in the main study). The results of confirmatory factor analysis showed that a two-factor orthogonal structure had the best fit $(\mathrm{CMIN}=52.580 ; \mathrm{df}=39 ; p=.072 ; \mathrm{GFI}=.971 ; \mathrm{CFI}=.957 ; \mathrm{RMSEA}=.034 ;$ Pclose $=.884)$. The high internal reliability of both scales ((Cronbach's $\alpha$ for "Promotion focus" scale 0.77 and for "Prevention focus" scale -0.78 ) and temporal stability (4 weeks interval) were shown. The number of questions is equal to the original version of the questionnaire. The overall relationship between promotion and prevention focuses and cognitive biases in decision making was partly shown, which stands for construct validity of the scales. The Russian version of Regulatory
\end{abstract}


Focus Questionnaire can be used in future research in samples aged 17 to 23 . At the end, the full text of the questionnaire, the instructions and scores calculation are listed.

Keywords: Regulatory Focus Questionnaire (RFQ), regulatory focus theory, promotion focus, prevention focus, achievement motivation.

\section{References}

Agafonov, A. Yu., \& Kozlov, D. D. (2014). Cognitive strategies in the work of consciousness and the unconscious. Izvestiya Samarskogo Nauchnogo Tsentra RAN, 16(2), 864-872. (in Russian)

Arkes, H. R., \& Blumer, C. (1985). The psychology of sunk cost. Organizational Behavior and Human Decision Processes, 35, 124-140.

Atkinson, J. W. (1964). An introduction to motivation. Princeton, NJ: Van Nostrand.

Bąk, W., Łaguna, M., \& Bondyra-Łuczka, E. (2015). Kwestionariuszowe metody pomiaru ukierunkowań regulacyjnych. Polskie adaptacje kwestionariuszy RFQ i RFS. Psychologia Społeczna, 10, 84-99. doi:10.7366/1896180020153206

Bentler, P. M. (1972). A lower method for the dimension-free measurement of internal consistency. Social Science Research, 1(4), 343-357. doi:10.1016/0049-089X(72)90082-8

Burlachuk, L. F. (2006). Psikhodiagnostika [Psychodiagnostics ]. Saint Petersburg: Piter. (in Russian)

Byrne, B. M. (2010). Structural equation modeling with AMOS: Basic concepts, applications, and programming (2nd ed.). New York: Taylor \& Francis Group.

Cesario, J., Corker, K. S., \& Jelinek, S. (2013). A self-regulatory framework for message framing. Journal of Experimental Social Psychology, 49(2), 238-249. doi:10.1016/j.jesp.2012.10.014

Crowe, E., \& Higgins, E. T. (1997). Regulatory focus and strategic inclinations: Promotion and prevention in decision-making. Organizational Behavior and Human Decision Processes, 69, 117-132. doi:10.1006/obhd.1996.2675

Förster, J., \& Higgins, E. T. (2005). How global versus local perception fits regulatory focus. Psychological Science, 16, 631-636. doi:10.1111/j.1467-9280.2005.01586.x

Förster, J., Higgins, E. T., \& Bianco, A. T. (2003). Speed/accuracy decisions in task performance: Builtin trade-off or separate strategic concerns? Organizational Behavior and Human Decision Processes, 90, 148-164. doi:10.1016/S0749-5978(02)00509-5

Friedman, R., \& Förster, J. (2001). The effects of promotion and prevention cues on creativity.Journal of Personality and Social Psychology, 81(6), 1001-1013. doi:10.1037//0022-3514.81.6.1001

Halvorson, H. G., \& Higgins, E. T. (2014). Psikhologiya motivatsii. Kak glubinnye ustanovki vliyayut na nashi zhelaniya i postupki [Psychology of motivation. How hidden attitudes influence our wishes and actions] Moscow: Mann, Ivanov, Ferber. (in Russian) (Trans. of: Halvorson, H. G., \& Higgins, E. T. (2013). Focus: Use different ways of seeing the world for success and influence. New York: Hudson Street Press.)

Heine, S. J., \& Ruby, M. B. (2010). Cultural psychology. Wiley Interdisciplinary Revieres: Cognitive Science, 1(2), 254-266. doi:10.1002/wcs.7

Higgins, E. T. (1997). Beyond pleasure and pain. American Psychologist, 52, 1280-1300. doi:10.1037/0003-066X.52.12.1280

Higgins, E. T. (1998). Promotion and prevention: Regulatory focus as a motivational principle. In M. P Zanna (Ed.), Advances in experimental social psychology (Vol. 30, pp. 1-46). New York: Academic Press. 
Higgins, E. T. (2000). Making a good decision: Value from fit. American Psychologist, 55, 1217-1230. doi:10.1037/0003-066X.55.1

Higgins, E. T. (2005). Value from regulatory fit. Current Directions in Psychological Science, 14, 209-213. doi:10.1111/j.0963-7214.2005.00366.x

Higgins, E. T. (2006). Value from hedonic experience and engagement. Psychological Review, 113(3), 439-460. doi:10.1037/0033- 295X. 113.3.439

Higgins, E. T., Friedman, R. S., Harlow, R. E., Idson, L. C., Ayduk, O. N., \& Taylor, A. (2001). Achievement orientations from subjective histories of success: Promotion pride versus prevention pride. European Journal of Social Psychology, 31, 3-23. doi:10.1002/ejsp.27

Higgins, E. T., Pierro, A., \& Kruglanski, A. W. (2008). Re-thinking culture and personality: How selfregulatory universal create cross-cultural differences. In R. M. Sorrentino \& S. Yamaguchi (Eds.), Handbook of motivation and cognition across cultures (pp. 161-190). New York: Elsevier.

Higgins, E. T., Roney, C., Crowe, E., \& Hymes, C. (1994). Ideal versus ought predilections for approach and avoidance: Distinct self-regulatory systems. Journal of Personality and Social Psychology, 66, 276-286. doi:10.1037/0022-3514.66.2.276

Higgins, E. T., Shah J., \& Friedman, R. S. (1997). Emotional responses to goal attainment: Strength of regulatory focus as moderator. Journal of Personality and Social Psychology, 72, 515-525. doi: 10.1037/0022-3514.72.3.515

Higgins, E. T., \& Silberman, I. (1998). Development of regulatory focus: Promotion and prevention as ways of living. In J. Heckhausen \& C. S. Dweck (Eds.), Motivation and self-regulation across the life span (pp. 78-113). New York: Cambridge University Press.

Higgins, E. T., \& Tykocinski, O. (1992). Self-discrepancies and biographical memory: Personality and cognition at the level of psychological situation. Personality and Social Psychology Bulletin, 18, 527-535. doi:10.1177/0146167292185002

Ip, G. W. M., \& Chiu, C. Y. (2001). Implicit theories and responses to achievement setbacks. In C. Y. Chiu, F. Salili, \& Y. Y. Hong (Eds.), Multiple competencies and self-regulated learning: Implications for multicultural education (pp. 193-204). Greenwich, CT: Information Age.

Jackson, D. N. (1974). The personality research form. Port Huron, MI: Research Psychologists Press.

Kacperski, C., \& Kutzner, F. L. W. (2016). Adherence to tactical choices mediates regulatory fit effects in table tennis. International Journal of Sport and Exercise Psychology, 1-9. Advance online publication. doi:10.1080/1612197X.2016.1199581

Kahneman, D., \& Tversky, A. (1979). Prospect theory: An analysis of decision under risk. Econometrica, 47, 263-291.

Kline, R. B. (2011). Principles and practice of structural equation modeling (3rd ed.). New York: The Guilford Press.

Kurginyan, S. S. (2013). Testing the Selves Questionnaire. Psychology. Journal of Higher School of Economics, 10(2), 122-137. (in Russian)

Kurman, J., \& Hui, C. (2011). Promotion, prevention or both: Regulatory focus and culture revisited. Online Readings in Psychology and Culture, 5(3). doi:10.9707/2307-0919.1109

Kutzner, F. L. W., Förderer, S., \& Plessner, H. (2013). Regulatory fit improves putting in top golfers. Sport, Exercise, and Performance Psychology, 2(2), 130-137. doi:10.1037/a0030733

Lalwani, A. K., Shrum, L. J., \& Chiu, C. Y. (2009). Motivated response styles: The role of cultural values, regulatory focus, and self-consciousness in socially desirable responding. Journal of Personality and Social Psychology, 96(4), 870-872. doi:10.1037/a0014622 
Lee, A. Y., Aaker, J. L., \& Gardner, W. L. (2000). The pleasures and pains of distinct self-construals: The role of interdependence in regulatory focus. Journal of Personality and Social Psychology, 78, 11221134. doi:10.1037/0022-3514.78.6.1122

Leikas, S., Lönnqvist, J.-E., Verkasalo, M., \& Lindeman, M. (2009). Regulatory focus systems and personal values. European Journal of Social Psychology, 39(3), 415-429. doi:10.1002/ejsp.547

Molden, D. (2012). Motivated strategies for judgment: How preferences for particular judgment processes can affect judgment outcomes. Social and Personality Psychology Compass, 6(2), 156169. doi:10.1111/j.1751-9004.2011.00424.x

Molden, D. C., Lee, A. Y., \& Higgins, E. T. (2008). Motivations for promotion and prevention. In J. Y. Shah, \& W. L. Gardner (Eds.), Handbook of motivation science (pp. 169-187). New York: Guilford Press.

Mowle, E. N., Georgia, E. J., Doss, B. D., \& Updegraff, J. A. (2014). Application of regulatory focus theory to search advertising. Journal of Consumer Marketing, 31(6/7), 494-502. doi:10.1108/JCM06-2014-1003

Nasledov, A. D. (2013). IBM SPSS 20 i AMOS: professional'nyi statisticheskii analiz dannykh [IBM SPSS 20 i AMOS: professional statistical data analysis]. Saint Petersburg: Piter. (in Russian)

Plessner, H., Unkelbach, C., Memmert, D., Baltes, A., \& Kolb, A. (2009). Regulatory fit as a determinant of sport performance: How to succeed in a soccer penalty-shooting. Psychology of Sport and Exercise, 10(1), 108-115. doi:10.1016/j.psychsport.2008.02.001

Rusu, A., Hojbotă, A., \& Sălăgean, N. (2015). Measuring chronic regulatory focus in Romania: adaptation of the Regulatory Focus Questionnaire. Romanian Journal of Applied Psychology, 17(2), 45-51.

Schwab, S., Wolf, O. T., \& Memmert, D. (2015). The influence of motivation on stress: is it stressful not to fit? Stress, 18(5), 597-601. doi:10.3109/10253890.2015.1053453

Shah, J., Higgins, E. T., \& Friedman, R. S. (1998). Performance incentives and means: How regulatory focus influences goal attainment. Journal of Personality and Social Psychology, 74, 285-293. doi:10.1037/0022-3514.74.2.285

Shapiro, A., \& ten Berge, J. M. F. (2000). The asymptotic bias of minimum trace factor analysis, with applications to the greatest lower bound to reliability. Psychometrika, 65(3), 413-425. doi:10.1007/BF02296154

Shmelev, A. G. (2013). Prakticheskaya testologiya. Testirovanie v obrazovanii, prikladnoi psikhologii i upravlenii personalom [Practical testology. Testing in education, applied psychology and human resource management]. Moscow: OOO "IPTs Maska".

Tanner, W. P. Jr., \& Swets, J. A. (1954). A decision-making theory of visual detection. Psychological Review, 61, 401-409. doi:10.1037/h0058700

Zhang, Y., \& Mittal, V. (2007). The attractiveness of enriched and impoverished options: Culture, selfconstrual, and regulatory focus. Personality and Social Psychology Bulletin, 33(4), 588-598. doi:10.1177/0146167206296954

Valeriia A. Gershkovich - associate professor, Department of the Problems of Convergence in Natural Sciences and Humanities, Saint Petersburg State University, Ph.D.

Research area: cognitive psychology, cognitive control in memory and learning tasks, choking under pressure, achievement motivation, memory illusions.

E-mail: valeria.gershkovich@gmail.com 
Nadezhda V. Moroshkina - senior research fellow, Laboratory of Cognitive Studies, Saint Petersburg State University, Ph.D.

Research area: cognitive psychology, implicit learning and implicit memory, consciousness and cognitive unconsciousness, frame effects, achievement motivation.

E-mail: moroshkina.n@gmail.com

Almara K. Kulieva - Ph.D. student, Department of General Psychology, Saint Petersburg State University.

Research area: cognitive psychology, consciousness, psychology of will, visual illusions.

E-mail: almara.kulieva@gmail.com

Andrey D. Nasledov - associate professor, Faculty of Psychology, Saint Petersburg State University, Ph.D.

Research area: application of mathematical methods and models in psychology; measurement methods in psychology; development of psychodiagnostic methods.

E-mail: andrey.nasledov@gmail.com 